\title{
Homeostatic Plasticity in the Visual Thalamus by Monocular Deprivation
}

\author{
Thomas E. Krahe and William Guido \\ Department of Anatomy and Neurobiology, Virginia Commonwealth University Medical Center, Richmond, Virginia 23298
}

\begin{abstract}
Monocular deprivation (MD) is a classic paradigm for experience-dependent cortical plasticity. One form is known as homeostatic plasticity, in which neurons innervated by the deprived eye show a remarkable capacity to compensate for degraded visual signals in an attempt to stabilize network activity. Although the evidence supporting homeostatic plasticity in visual cortex is extensive, it remains unclear whether neurons in subcortical visual structures respond to MD in a similar manner. Here we examined whether cells in the dorsal lateral geniculate nucleus (dLGN), the thalamic relay between the retina and visual cortex, show similar forms of experiencedependent homeostatic plasticity following MD. Two-week-old mice were monocularly deprived for a period of 5-7 d and miniature EPSCs (mEPSCs) were obtained from cells located in dLGN regions receiving input from the deprived or nondeprived eye. We found that MD promotes increases in the frequency and amplitude of mEPSCs and were restricted to the monocular segment contralateral to the deprived eye. These changes were accompanied by an increase in the probability of glutamate release at corticothalamic terminals that arise from the deprived visual cortex. Our findings indicate that homeostatic synaptic regulation from MD extends beyond cortical circuitry and shed light on how the brain modulates and integrates activity in the face of altered sensory experience.
\end{abstract}

\section{Introduction}

The formation of precise neuronal connections relies on the nature of experience early in life. In the case of the visual system, closing one eye [monocular deprivation (MD)] for a few days leads to profound changes in cortical circuitry and synaptic transmission (Hubel and Wiesel, 1970; Olson and Freeman, 1980; Antonini et al., 1999). Considerable effort has been directed toward defining these changes in terms of the competition between the two eyes (Gordon and Stryker, 1996; Smith and Trachtenberg, 2007; Smith et al., 2009). However, evidence also shows that neurons receiving input solely from the deprived eye possess a remarkable capacity to adjust to the loss of patterned vision. In such cases, the visual responsiveness of deprived neurons is enhanced by increases in synaptic strength, intrinsic excitability, or both (Turrigiano and Nelson, 2004; Turrigiano, 2008). For instance, brief periods of MD after eye opening can increase the amplitude of AMPA-mediated miniature EPSCs (mEPSC) in a layer- and age-dependent manner (Desai et al., 2002; Maffei et al., 2004; Maffei and Turrigiano, 2008). Similar results have been reported using in vivo calcium imaging showing that cells driven exclusively by the deprived eye increase their visual responsiveness following MD (Mrsic-Flogel et al., 2007). This ability to

\footnotetext{
Received March 7, 2011; accepted March 22, 2011.

Author contributions: T.K. and W.G. designed research; T.K. and W.G. performed research; T.K. analyzed data; T.K. and W.G. wrote the paper.

This work was supported by National Institutes of Health R01 Grant EY12716 (to W.G.). We thank R. El-danaf for histological assistance and E. K. Dilger for a critical reading of the manuscript.

Correspondence should be addressed to William Guido, Departmentt of Anatomy and Neurobiology, Virginia Commonwealth University Medical Center, Sanger Hall, 1101 East Marshall Street, Richmond, VA 23298. E-mail: wguido@vcu.edu.

DOI:10.1523/JNEUROSCI.1173-11.2011

Copyright $\odot 2011$ the authors $\quad 0270-6474 / 11 / 316842-08 \$ 15.00 / 0$
}

compensate for decreased sensory input to maintain network stability is a hallmark feature of cortical circuits (Turrigiano and Nelson, 2004; Turrigiano, 2008). Whether such forms of plasticity are found in subcortical sensory structures remains largely untested.

Compared with the visual cortex, evidence that alterations in pattern vision can induce plastic changes in subcortical circuitry is far more limited. This may not be too surprising since the majority of changes in neural connectivity are seen before eye opening, when retinal waves are the predominant form of visual activity (Demas et al., 2003; Torborg and Feller, 2005; Huberman et al., 2008). However, recent findings have shown that abnormal visual activity influences the strength and final patterns of connectivity in the visual sector of the dorsal thalamus, the lateral geniculate nucleus (dLGN). The persistence of abnormal spontaneous activity after eye opening causes newly refined eye-specific domains to desegregate and return to a diffuse state (Demas et al., 2006). Dark rearing for a few days during the same period weakens retinogeniculate synaptic strength and increases the number of retinal inputs converging on single dLGN cells (Hooks and Chen, 2006, 2008). Interestingly, the timing of these events coincides with the homeostatic plasticity reported in the visual cortex after MD (Desai et al., 2002; Maffei et al., 2004; Maffei and Turrigiano, 2008). Nonetheless, it is unclear whether the dLGN shares a similar form of plasticity. To address this, we investigated whether MD promotes homeostatic synaptic changes in dLGN cells of the mouse.

\section{Materials and Methods}

We used wild-type C57BL/6 mice that were obtained from timepregnant females acquired from commercial vendors. All procedures were performed in compliance with the Institutional Animal Care and Use Committee at Virginia Commonwealth University. 


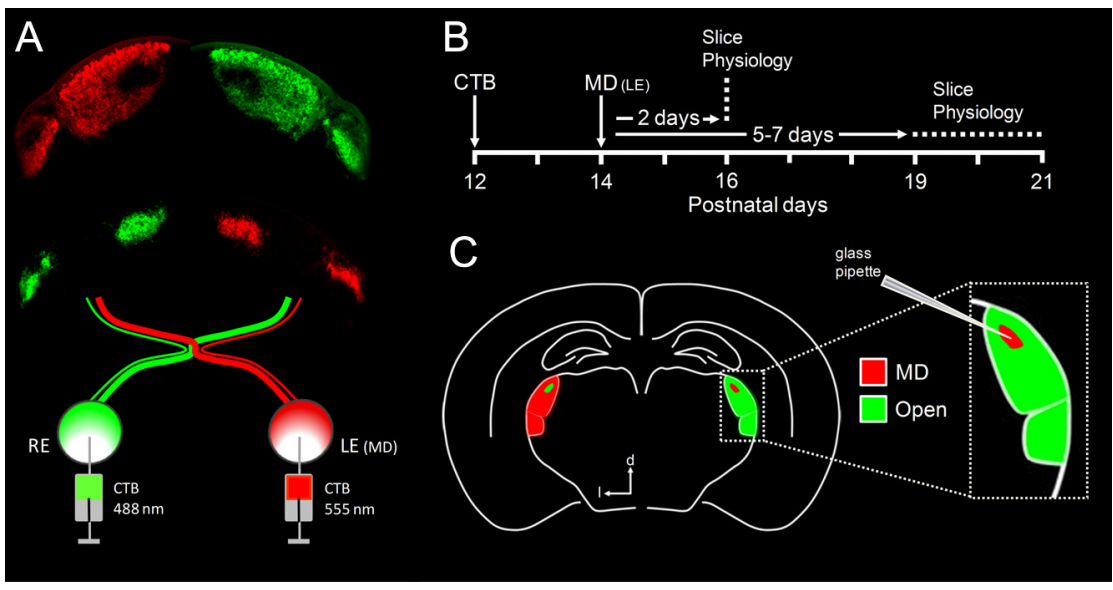

Figure 1. Experimental design. $A$, Schematic of anterograde labeling of retinogeniculate projections with CTB conjugated to different Alexa dyes. $\boldsymbol{B}$, Timeline showing when CTB injections, MD, and recordings occurred. $\boldsymbol{C}$, Schematic showing a coronal slice of CTB-labeled projections in dLGN. The inset depicts pipette placement in the region of dLGN receiving input from the ipsilateral, monocularly deprived eye. LE, Left eye; RE, right eye; d, dorsal; l, lateral.

Anterograde labeling of retinogeniculate projections. At postnatal day (P)12, mice were deeply anesthetized with isoflurane, the sclera and cornea were pierced with a sharp-tipped glass pipette, and excess vitreous was drained. Another pipette, containing a $1 \%$ solution of cholera toxin B subunit (CTB) (Invitrogen) conjugated to either Alexa Fluor 488 (green) or Alexa Fluor 555 (red) dissolved in distilled water, was attached to a picospritzer and the solution was injected into one eye (Jaubert-Miazza et al., 2005). Each eye was injected with a different fluorescent conjugate so the terminal fields from both eyes could be visualized in the same slice during in vitro whole-cell recordings.

Monocular deprivation. Two days after CTB injections (P14), animals were deeply anesthetized with isoflurane and the eyelid of the left eye was sutured closed. Animals were inspected daily for openings in sutured eyelids or for signs of infection, and those with such problems were excluded from the present study. After 2 or 5-7 d of MD, in vitro wholecell recordings were done in acutely prepared thalamic slices.

Preparation of thalamic slices. Slices were prepared in a manner similar to previous reports (Bickford et al., 2010; Dilger et al., 2011). Animals were deeply anesthetized with isoflurane and decapitated. The brain was removed rapidly and immersed in an oxygenated $4^{\circ} \mathrm{C}$ sucrose solution containing the following (in mM): $28 \mathrm{NaHCO}_{3}, 23$ sucrose, $11 \mathrm{MgSO}_{4}$, 0.11 glucose, $2.75 \mathrm{KCl}, 1.4 \mathrm{NaH}_{2} \mathrm{PO}_{4}, 0.5 \mathrm{CaCl}_{2}$. A block of tissue containing the dLGN was submerged in oxygenated $4^{\circ} \mathrm{C}$ sucrose solution and sectioned with a vibratome (Leica VT1000S) in the coronal or parasaggital plane at a thickness of $300 \mu \mathrm{m}$. Slices containing the dLGN were placed in a holding chamber and incubated with artificial CSF (ACSF; in mM: $124 \mathrm{NaCl}, 2.5 \mathrm{KCl}, 1.25 \mathrm{NaH}_{2} \mathrm{PO}_{4}, 2.0 \mathrm{MgSO}_{4}, 26 \mathrm{NaHCO}_{3}, 10$ glucose, $2 \mathrm{CaCl}_{2}$, saturated with $95 \% \mathrm{O}_{2} / 5 \% \mathrm{CO}_{2}, \mathrm{pH} 7.4$ ) at $30-35^{\circ} \mathrm{C}$ for $25 \mathrm{~min}$ and then rested at room temperature for at least $35 \mathrm{~min}$. Individual slices were then transferred to a recording chamber maintained at $32^{\circ} \mathrm{C}$ and perfused continuously at a rate of $2.0 \mathrm{ml} / \mathrm{min}$ with oxygenated ACSF.

Recording procedures. In vitro recordings were done with the aid of a fixed-stage microscope (Olympus BX51WI) equipped with differential interference contrast optics and water-immersion objectives to view individual neurons within the slice. Patch electrodes were pulled vertically in two stages from borosilicate glass and filled with a solution containing the following (in mM): $71.5 \mathrm{~K}$-gluconate, $5.7 \mathrm{HEPES}, 2 \mathrm{MgATP}, 0.3 \mathrm{NaCl}$, $0.1 \mathrm{NaGTP}$, and $1 \%$ biocytin ( $\mathrm{pH} 7.25,260 \mathrm{osmol} / \mathrm{L}$ ). The final tip resistance of filled electrodes was 3-6 M $\Omega$. Whole-cell recordings were done in voltage-clamp mode and AMPA-mediated mEPSCs were recorded at a holding potential of $-70 \mathrm{mV}$ and in the presence of tetrodotoxin (TTX, $1 \mu \mathrm{M}$; Tocris Bioscience) and the $\mathrm{GABA}_{\mathrm{A}}$ receptor antagonist bicuculline (BIC, $25 \mu \mathrm{M}$; Tocris Bioscience). Relay dLGN neurons were identified by their soma size, resting membrane potential, and, in some cases, their intrinsic membrane properties and dendritic morphology. Pipette capac- itance, series resistance, and whole-cell capacitance were carefully monitored and compensated electronically during the recording. All cells had a resting membrane potential that was between -60 and $75 \mathrm{mV}$, a series resistance between 3 and $6 \mathrm{M} \Omega$, and input resistance $>200 \mathrm{M} \Omega$. We did not observe any significant differences in the average $( \pm \mathrm{SE})$ input resistance between control (normally reared), deprived [contralateral deprived eye (CDE), ipsilateral deprived eye (IDE)], and open [contralateral open eye (COE), ipsilateral open eye (IOE)] eye cells (control, $308.89 \pm 25.13$; CDE, $315.98 \pm 15.04 ;$ IDE, $274.54 \pm 16.20 ; \mathrm{COE}$, $247.62 \pm 7.11$; IOE, $288.0 \pm 36.76 \mathrm{M} \Omega$; oneway ANOVA, $F=1.96, p=1.24$ ).

Events were recorded for $5 \mathrm{~min}$ and a maximum of 350 events were analyzed per neuron. Groups were assigned based on whether the neuron was recorded in a region of dLGN that received input from the contralateral or ipsilateral deprived or nondeprived eye. All neuronal activity was recorded with an Axopatch-1D (Axon Instruments) or a 2400 A-M Systems amplifier, digitized at $20 \mathrm{kHz}$ using an interface unit (BNC-2090; National Instruments), and stored on a computer. Current traces were filtered at $5 \mathrm{kHz}$; events were detected and amplitudes measured using WinEDR (Windows Electrophysiology Disk Recorder v3.8.2; Strathclyde Electrophysology Software). The threshold for detecting mEPSCs was set at two times the root mean square (RMS; $2.90 \pm 0.05 \mathrm{pA}$ ). There was no significant difference in RMS noise among any of the experimental groups (one-way ANOVA, $F=0.281, p=0.84$ ). Measured mEPSCs also had rise times that were $<2 \mathrm{~ms}$ (measured between 10 and $90 \%$ of amplitude).

After recording, slices were fixed overnight with $4 \%$ paraformaldehyde in $0.1 \mathrm{M}$ PBS, then washed with a $0.1 \%$ solution of Alexa Fluor 647 conjugated to streptavidin dissolved in PBS with $0.1 \%$ Triton X-100. Slices were mounted with Prolong (Invitrogen), cured overnight at room temperature, and stored in a freezer at $-20^{\circ} \mathrm{C}$. Labeled sections were visualized with an upright epifluorescence microscope (Nikon E600) to confirm the location and morphology of the labeled cells.

Synaptic stimulation. Synaptic responses were evoked by electrical stimulation of the optic tract (OT) or corticothalamic (CT) axons using bipolar sharpened tungsten electrodes (0.5 M 2 ; A-M Systems). For separate and independent stimulation of OT and CT, experiments were performed using parasagittal slices (Turner and Salt, 1998). Bipolar electrodes were positioned on the internal capsule for stimulation of CT fibers and at the caudal ventral border of the dLGN for OT stimulation (Turner and Salt, 1998; Govindaiah and Cox, 2004). Stimulating electrodes were $0.5-1 \mathrm{~mm}$ away from the dLGN border. Whole-cell recordings were done in voltage-clamp mode at a holding potential of $-70 \mathrm{mV}$ and in the presence of BIC $(25 \mu \mathrm{M})$ and the $\mathrm{GABA}_{\mathrm{B}}$ receptor antagonist 3-minopropyl diethoxymethyl phosphinic acid (10 $\mu \mathrm{M}$; Tocris Bioscience). Initially, single synaptic responses evoked once every $20 \mathrm{~s}$ across a range of stimulus intensities $(0.1-0.3 \mathrm{~ms}, 0.1-1 \mathrm{~mA})$ were obtained and the stimulus producing the maximal response was used for a paired-pulse protocol. Since CT stimulation gives rise to graded responses, and to minimize such variability, it was necessary to choose a stimulus intensity that recruited as many fibers as possible (Granseth et al., 2002; Granseth and Lindström, 2003).

Paired stimuli were delivered every $30 \mathrm{~s}$ at a rate of $10 \mathrm{~Hz}(100 \mathrm{~ms}$ interpulse interval). Previous reports indicate that these parameters are near optimal for evoking the peak of both paired-pulse facilitation and depression at the corticothalamic and retinogeniculate synapses, respectively (Turner and Salt, 1998; Granseth et al., 2002; Chen and Regehr, 2003; Granseth and Lindström, 2003; Govindaiah and Cox, 2004; Alexander et al., 2006). Recordings were limited to dLGN regions corresponding to the monocular segment of dLGN (Coleman et al., 2009). 


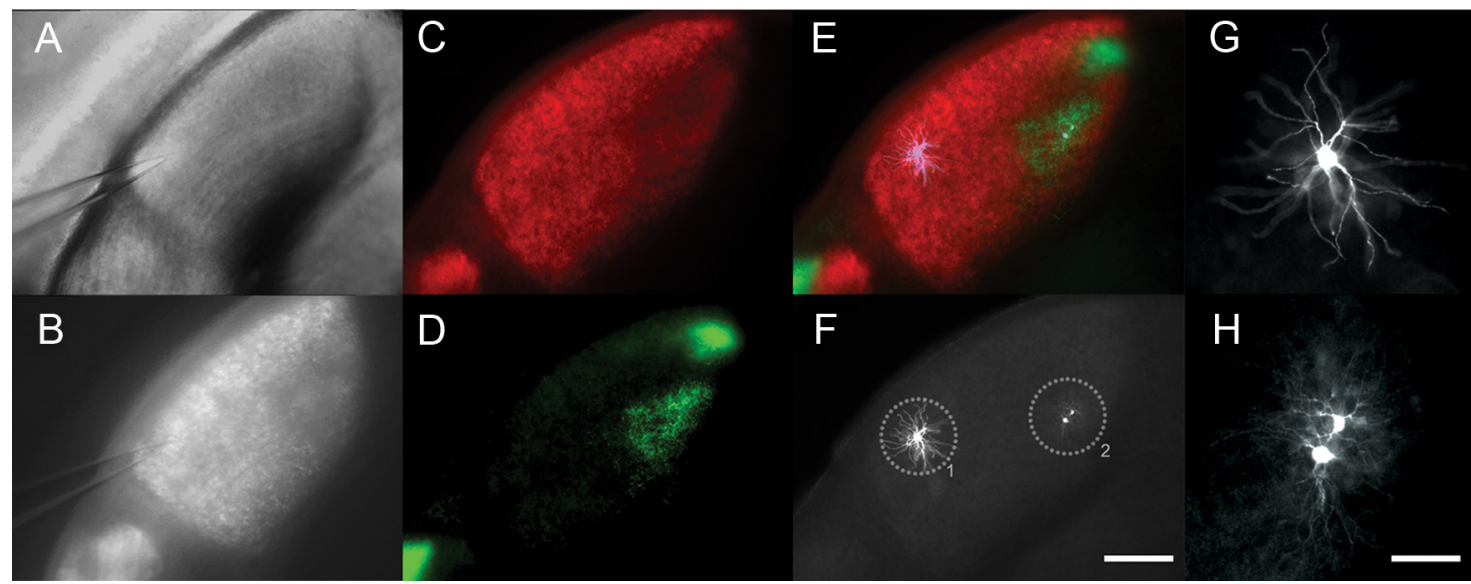

Figure 2. Visualization of labeled retinal projections and biocytin-filled relay cells in $\mathrm{dLGN} . \boldsymbol{A}, \boldsymbol{B}$, Differential interference contrast $(\boldsymbol{A})$ and fluorescence $(\boldsymbol{B})$ images taken through a microscope during in vitro dLGN slice recording. $\boldsymbol{A}$, Patch pipette filled with internal solution and biocytin. $\boldsymbol{B}$, (TB-labeled terminal fields arising from the contralateral deprived eye. $\boldsymbol{C}-\boldsymbol{F}$, Same section, fixed and photographed through a fluorescent microscope depicting terminal fields from the contralateral $(\boldsymbol{C}$, red) and ipsilateral ( $\boldsymbol{D}$, green) eyes, the superimposed fluorescence pattern $(\boldsymbol{E})$, and highlighted biocytin-filled relay neurons $(\boldsymbol{F}) . \boldsymbol{G}, \boldsymbol{H}$, High-power views of cells in dashed circles $1(\boldsymbol{G})$ and 2 ( $\boldsymbol{H}$ ) from $\boldsymbol{F}$. Scale bars: $\boldsymbol{A}-\boldsymbol{F}$ (in $\boldsymbol{F}), 200 \mu \mathrm{m} ; \boldsymbol{G}, \boldsymbol{H}$ (in $\boldsymbol{H}), 20 \mu \mathrm{m}$.

\section{Results}

To study the effects of visual deprivation in dLGN, we monocularly deprived 2-week-old mice for a period of 5-7 d and measured mEPSCs of neurons located in dLGN regions receiving contralateral or ipsilateral input from the deprived or nondeprived eye. To distinguish these regions, eye injections of the fluorescent anterograde tracer CTB were made to visualize eye-specific domains during in vitro recordings (Fig. 1). Figure 2 shows an example of a thalamic slice preparation, illustrating the location of recorded dLGN cells in relation to anterogradely labeled retinal terminal fields. Retinal projections from the two eyes are already segregated into non-overlapping eye-specific domains by the time of natural eye opening (Jaubert-Miazza et al., 2005; Demas et al., 2006). Therefore, cells within these regions receive monocular input, which allows one to assess the effects of MD in the absence of competitive interactions between the two eyes. Moreover, recordings done in normally reared mice indicate that mEPSCs from cells located within contralateral and ipsilateral eye regions are not different from each other (Fig. 3). Finally, mEPSCs from normally reared mice were similar in strength and frequency across the postnatal ages studied (P16 vs P19-P21, $21.05 \pm 1.55$ vs $22.33 \pm$ $0.86 \mathrm{pA}$, one-way ANOVA, $F=0.36, p=$ $0.55 ; 2.90 \pm 0.86$ vs $4.68 \pm 0.42$ events/s, one-way ANOVA, $F=2.85, p=0.1 ; \mathrm{P} 16$, $n=6$ cells from two animals; P19-P21, $n=33$ cells from five animals) (Figs. $4 C$, $5 D$, dashed lines).

Five to seven days of MD increased the amplitude of mEPSCs in the CDE region by $\sim 50 \%$ and mEPSC frequency by $180 \%$ relative to the IOE region $(26.55 \pm 1.66$ vs $18.22 \pm 1.89 \mathrm{pA}$ and

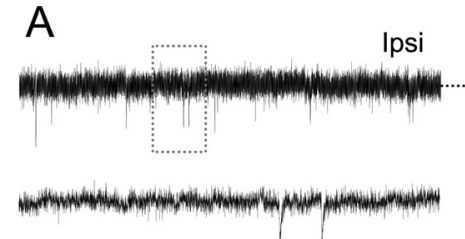

B
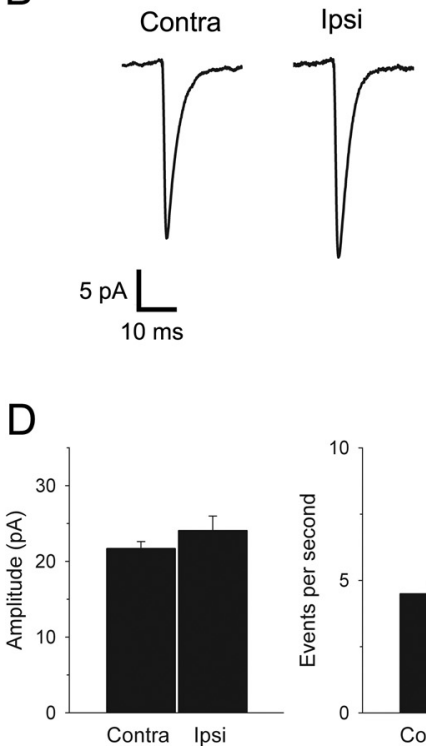
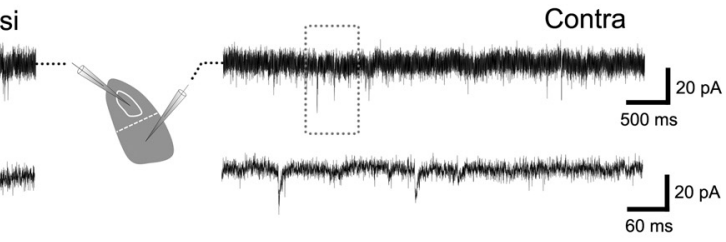

C
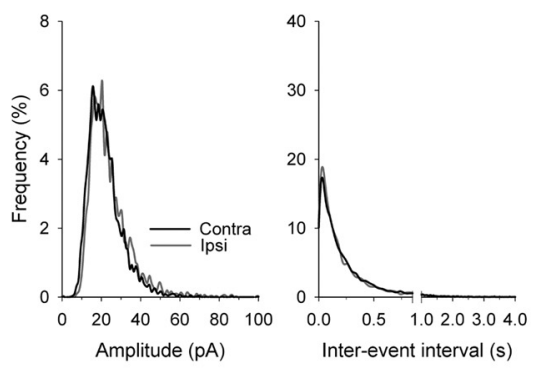

Figure 3. $m E P S C$ amplitude and frequency of normally reared mice. $\boldsymbol{A}$, Representative whole-cell voltage-clamp $m E$ EPSC recorded from two relay cells in dLGN regions receiving input from the contralateral (Contra) or ipsilateral (Ipsi) eye. The accompanying diagram depicts target regions. The mEPSCs were recorded between P19-P21 in TTX (1 $\mu \mathrm{M})$ and BIC (25 $\mu \mathrm{m})$ at a holding potential of $-70 \mathrm{mV}$. Highlighted sections are expanded in time below each trace. $\boldsymbol{B}$, Average mEPSCs recorded from contralateral and ipsilateral eye regions. $C$, Frequency histogram plots of $\mathrm{mEPSC}$ amplitude (left) and frequency (right) for contralateral (black) and ipsilateral (gray) eye regions. D, Group-averaged mEPSC amplitude (left) and frequency (right). Error bars depict SE. No significant differences were observed in mEPSCs amplitude or frequencies ( $t$ test, $p>0.2$, for both comparisons). Contralateral, $n=24$ cells; ipsilateral, $n=9$ cells; from five animals.

$13.38 \pm 1.88$ vs $4.76 \pm 0.98$ events/s, respectively; one-way ANOVAs, $F=4.357$ for amplitude, $F=11.710$ for frequency, Tamhane post hoc test, $p<0.05$ and $<0.01$, respectively; CDE, $n=28$ cells; IOE, $n=12$ cells, from eight animals) (Fig. $4 A, C$ ). Such 

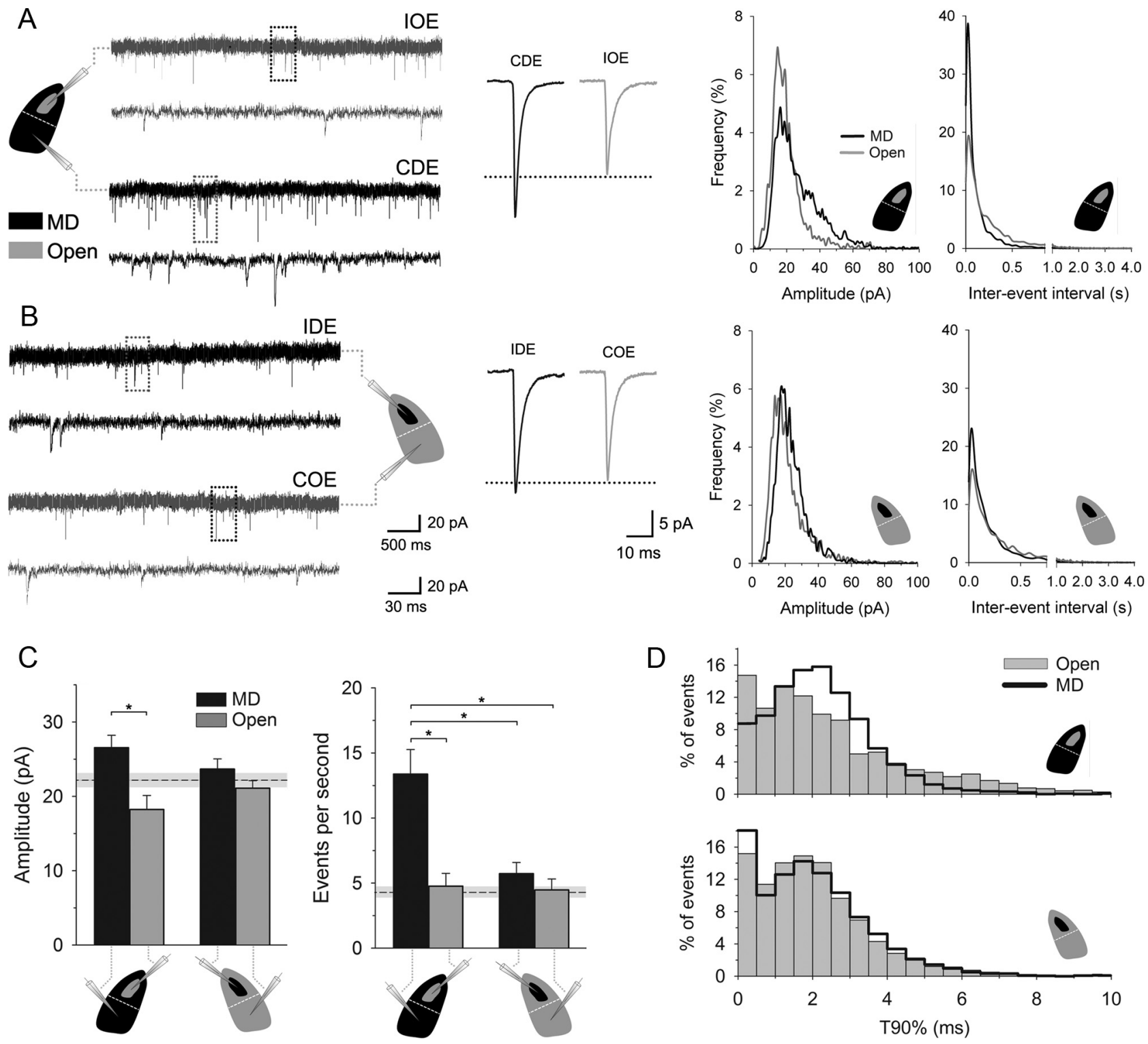

Figure 4. MD leads to an increase in mEPSC amplitude and frequency. $A, B$, Left, Representative whole-cell voltage-clamp mEPSCs recorded from four relay neurons in contralateral and ipsilateral, deprived (MD, black) and nondeprived (open, gray) eye regions (CDE, COE, IDE, and IOE) of a monocularly deprived (7 d) mouse at P14. The accompanying diagrams depict deprived and nondeprived targeted regions. The mEPSCs were recorded in TTX $(1 \mu \mathrm{M})$ and BIC $(25 \mu \mathrm{M})$ at a holding potential of $-70 \mathrm{mV}$. Highlighted sections are expanded in time below each trace. Middle, Average $\mathrm{mEPSCs}$ recorded from CDE, COE, IDE, and IOE regions. Amplitude was increased in CDE regions (black top trace). Right, Frequency histogram plots of mEPSC amplitude (left) and frequency (right). Deprivation shifted the distribution of mEPSCs in CDE regions toward larger amplitude and smaller interevent interval values (top). C, Group-averaged mEPSC amplitude (left) and frequency (right) after MD. Error bars depict SE. Dashed lines show the average amplitude and frequency of events recorded in age-matched normally reared mice (Fig. 3). Shaded area is \pm SE. MD significantly increased the mEPSC amplitude (left, ${ }^{*} p<0.05$ ) and frequency (right, ${ }^{*} p<0.01$ for all comparisons) of mEPSCs in CDE regions. CDE, $n=28$ cells; IOE, $n=12$ cells; COE, $n=22$ cells; IDE, $n=15$ cells. $D$, Histograms plot the duration (time from maximal to $10 \%$ of peak amplitude, bin width $=0.5 \mathrm{~ms}$ ) of mEPSCs for CDE and IOE (top) and COE and IDE regions (bottom).

differences were not observed in the opposite hemisphere when comparing mEPSCs in IDE against those from COE regions $(23.66 \pm 1.66$ vs $21.01 \pm 1.37 \mathrm{pA}$ and $5.72 \pm 0.84$ vs $4.47 \pm 0.86$ events/s, respectively; Tamhane post hoc test, $p>0.55$ for both comparisons; COE, $n=22$ cells; IDE, $n=15$ cells, from eight animals) (Fig. 4B, C). Moreover, CDE and IOE amplitudes were not significantly different from age-matched controls (Tamhane post hoc test, $p>0.26$ for both comparisons) (Fig. $4 C$, dashed line). These results indicate that MD modifies glutamatergic transmission in the monocular segment of dLGN (Coleman et al., 2009). The fact that the increase in mEPSC amplitude in this region is coupled with a concomitant increase in mEPSC frequency suggests that such compensation is driven by homeostatic changes in visual circuitry, rather than the induction of synaptic scaling per se (Turrigiano et al., 1998; Desai et al., 2002).

Relay cells in the dLGN receive glutamatergic inputs from two sources, layer VI cortical neurons and retinal ganglion cells (Sherman and Guillery, 2002). Corticothalamic synapses are concentrated on distal dendrites of relay neurons (Liu et al., 1998; Jones, 2002) and, as a consequence of dendritic filtering (Williams and Mitchell, 2008), corticothalamic postsynaptic events recorded somatically are reported to be slower (Golshani et al., 1998; Alexander et al., 2006). Thus, an increase in the number of slower mEPSCs in the CDE region following MD may indicate that the source of the observed changes in mEPSC frequency arise from corticothalamic inputs. In fact, the analysis of mEPSCs distribu- 
A

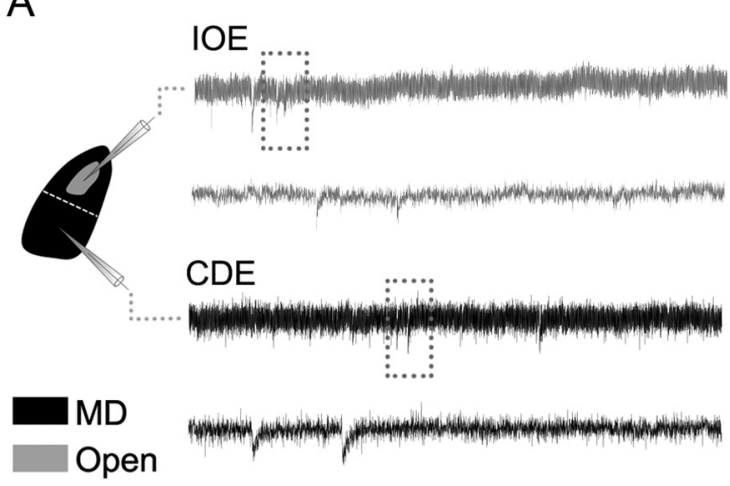

B
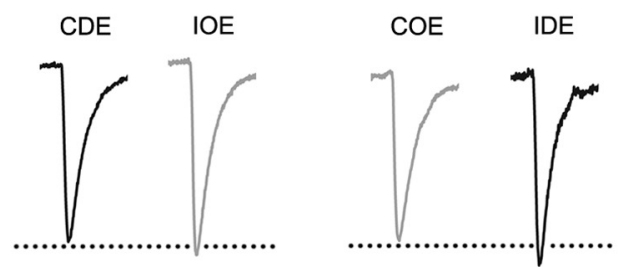

$\underset{10 \mathrm{~ms}}{\mathrm{fpA}}$
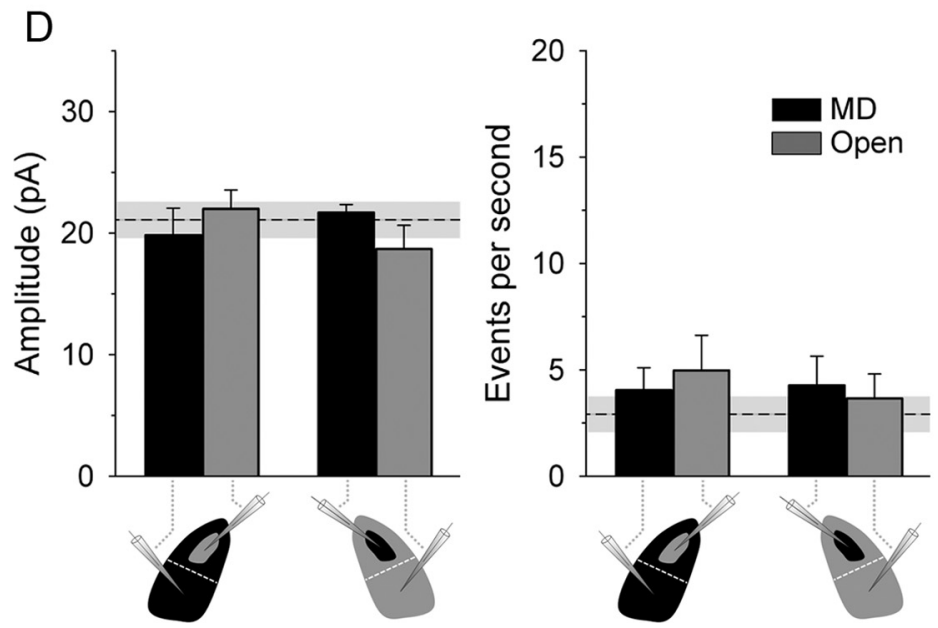

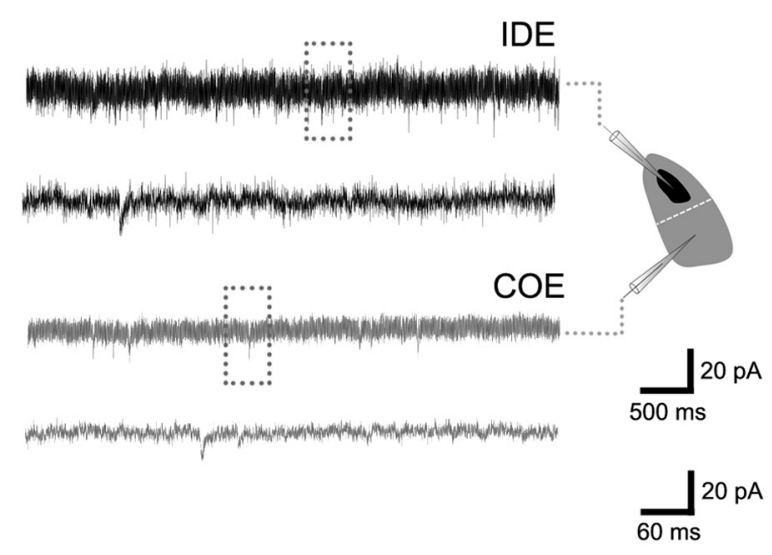

C
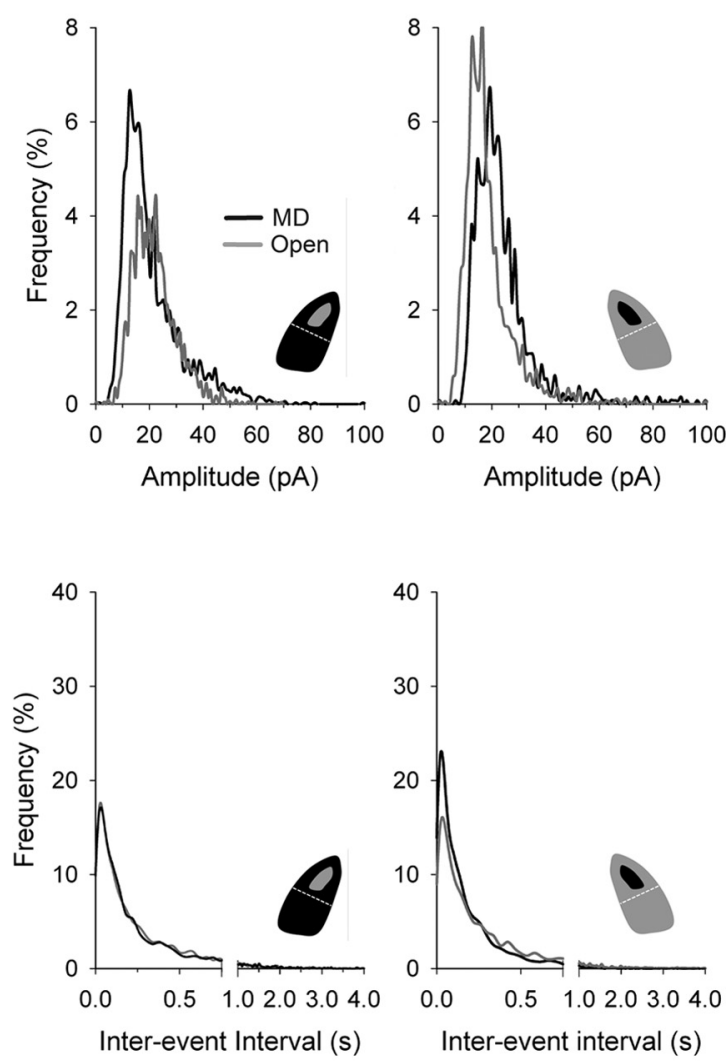

Figure 5. mEPSC amplitude and frequency after $2 \mathrm{~d}$ of MD. A, Representative whole-cell voltage-clamp mEPSCs recorded from four relay neurons in dLGN regions receiving input from the contralateral or ipsilateral deprived (MD, black) or nondeprived (open, gray) eye of a monocularly deprived (2d) mouse at P14. Diagrams and designations are the same as in Figure 4. Highlighted sections are expanded in time below each trace. mEPSCs were recorded in TTX (1 $\mu \mathrm{M})$ and BIC $(25 \mu \mathrm{M})$ at a holding potential of $-70 \mathrm{mV}$. B, Average mEPSCs recorded from contralateral and ipsilateral, deprived (black) and nondeprived (gray) eye regions. C, Frequency histogram plots of mEPSC amplitude (top) and frequency (bottom). D, Group-averaged mEPSC amplitude (left) and interevent interval (right) after MD. Error bars represent SE. Dashed lines show the average amplitude and frequency of events recorded in age-matched normally reared animals. Shaded area is \pm SE. Two days of MD did not significantly alter the amplitude or frequency of mEPSCs and were not different from age-matched normally reared animals (one-way ANOVAs, $F=0.620$ for amplitude, $F=0.341$ for frequency, $p>0.65$ for both variables). CDE, $n=15$ cells; IOE, $n=8$ cells; COE, $n=6$ cells; IDE, $n=5$ cells; from five animals. Normally reared, $n=6$ cells from two animals.

tions based on the time from maximal to $10 \%$ of peak amplitude revealed that the CDE region contains more slower events than the IOE region does (Wilcoxon signed rank test, $z=-2.035, p<$ 0.05 ; CDE, $n=8587$ events; IDE, $n=3360$ events) (Fig. $4 D$ ). By contrast, the same analysis comparing COE and IDE regions revealed no differences in decay times (Wilcoxon signed rank test, $z=-1.476, p=0.14$; COE, $n=4207$ events; IDE, $n=3945$ ) (Fig. $4 D)$. Overall, these results suggest that the increase in mEPSC frequency observed in the $\mathrm{CDE}$ region is of cortical origin.
Homeostatic plasticity in the visual cortex is seen after $2 \mathrm{~d}$ of MD (Desai et al., 2002, Maffei et al., 2004), suggesting that cortical synaptic modifications may occur faster than what we observed in dLGN. Figure 5 shows that $2 \mathrm{~d}$ of MD did not yield significant changes in mEPSC amplitude or frequency when deprived and nondeprived regions were compared. Indeed, these values did not differ from age-matched controls (Fig. 5D, dashed lines). This finding, together with the observed increases in the number of slower mEPSCs occurring in CDE region (Fig. $4 D$ ), 
A
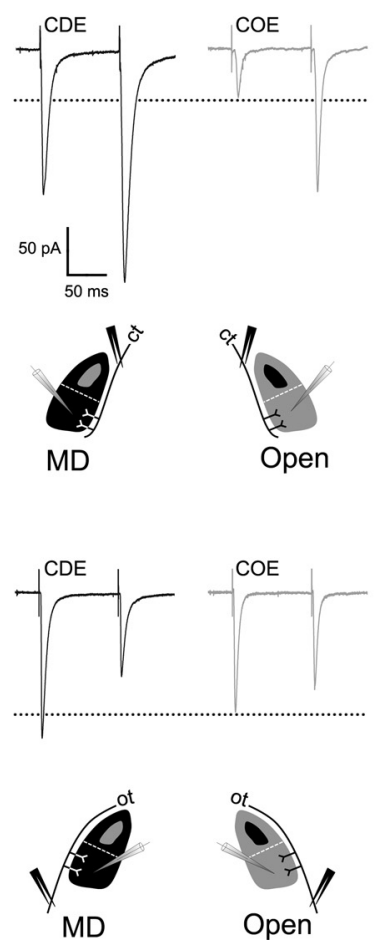

B
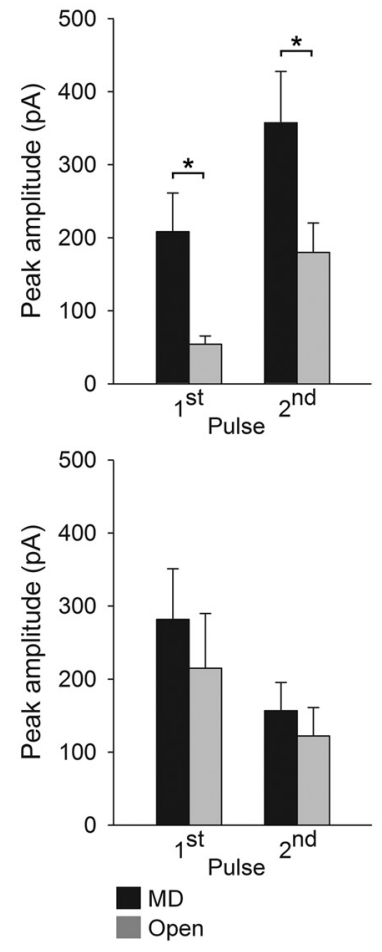

C
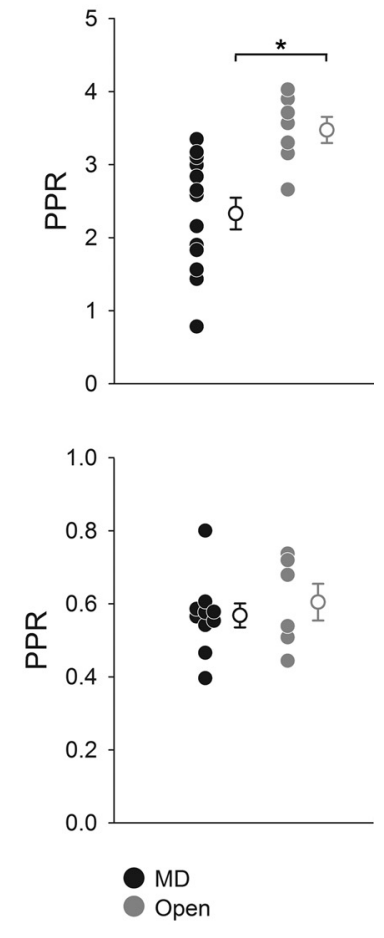

Figure 6. MD enhances corticothalamic glutamate release. $A$, Averaged eEPSCs ( $n=20$ per neuron) evoked by paired-pulse stimulation (100 ms interstimulus interval) of either CT (top) or 0T (bottom) projections in representative relay neurons located in deprived (black traces) and nondeprived (gray traces) eye regions. The accompanying diagrams depict location of stimulation and recording regions. $\boldsymbol{B}$, Averages of eEPSCs peak amplitude for the first and second responses evoked by CT (top) and OT (bottom) stimulation. For CT, eEPSC amplitudes recorded in deprived regions were significantly larger than in nondeprived ones ( $t$ test, ${ }^{*} p<$ 0.05 for both comparisons). Error bars represent SE. C, Paired-pulse ratios of CT (top) and OT (bottom) eEPSCs for deprived and nondeprived regions. Filled circles represent the mean PPR response of a single neuron. Open circles are the averaged PPR across cells. For CT, the averaged PPR in deprived neurons was significantly smaller than in nondeprived ones ( $t$ test, $\left.{ }^{*} p<0.01\right)$. Error bars represent \pm SE. CT MD, $n=13$ cells; CT open, $n=7 ;$ OT MD, $n=10 ; 0$ T open, $n=6$; from four animals.

suggests that homeostatic modifications first take place in the visual cortex, which, in turn, lead to an increase in the release of glutamate at corticothalamic terminals.

To confirm that MD leads to such an increase, we studied the release probability of glutamate at corticothalamic and retinothalamic terminals in monocularly deprived mice. A paired-pulse stimulation paradigm was used to activate CT and OT axons while recording the evoked synaptic responses in $\mathrm{CDE}$ and $\mathrm{COE}$ regions. As shown in Figure $6 \mathrm{~A}$, responses evoked by CT stimulation led to facilitation, whereas those by OT produced depression (Turner and Salt, 1998; Granseth et al., 2002; Chen and Regehr, 2003; Govindaiah and Cox, 2004). However, the amplitudes of the CT-evoked (e)EPSCs in the CDE region were significantly larger compared with those recorded in the COE region of the opposite hemisphere (Fig. $6 \mathrm{~B}$, top). This increase was evident across a wide range of stimulus intensities (CDE vs COE, 0.1-0.3 $\mathrm{mA}, 61.18 \pm 4.81$ vs $23.08 \pm 2.75 \mathrm{pA} ; 0.4-0.6 \mathrm{~mA}, 94.65 \pm 7.20 \mathrm{vs}$ $50.98 \pm 2.07 ; 0.7-0.9 \mathrm{~mA}, 138.44 \pm 10.44$ vs $77.28 \pm 3.67 \mathrm{pA}$; univariate ANOVA, $F=19.77$, Tamhane post hoc test, $p<0.001$ for all comparisons; $\mathrm{CDE}, n=553$ responses from eight to 10 cells; COE, $n=309$ responses from five cells). In contrast, no differences in eEPSC amplitudes were observed when OT was stimulated (Fig. 6B, bottom). Moreover, paired-pulse ratios (PPR; second eEPSC/ first eEPSC) for CT stimulation in the CDE region were significantly smaller than those in the COE region (Fig. 6C, top). Although the amplitude of the first eEPSC accounted for
$33 \pm 2.5 \%$ of the total (first plus second eEPSC) in the CDE region, this value was only $23 \pm 1.0 \%$ in the nondeprived dLGN. PPRs for OT stimulation yielded no significant differences (Fig. 6C, bottom). Thus, the reduced PPR, coupled with the increase in frequency and duration of mEPSCs (Fig. 4C,D), suggests that $\mathrm{MD}$ leads to an increased release of glutamate in CT terminals that innervate the CDE region of the dLGN.

Finally, it is worth noting whether the input/output relations associated with electrical stimulation of CT and OT afferents were preserved after MD. For example, it is well known that a progressive increase in CT stimulus intensity gives rise to graded responses, indicating that dLGN cells receive convergent input from several individual corticogeniculate cells (Turner and Salt, 1998; Granseth et al., 2002; Granseth and Lindström, 2003; Li et al., 2003). As shown in Figure 7, our results are consistent with these reports and further reveal that these relations are unaffected by MD. Following MD, response amplitude rose steadily with increases in stimulus intensity. MD also had no effect on the input/output relations associated with OT stimulation (Fig. 7). In both normal and deprived cases, a progressive increase in stimulus intensity gave rise to step-like increases in amplitude, indicating that dLGN cells at these ages receive input from just a few retinal ganglion cells (Chen and Regehr, 2000; Jaubert-Miazza et al., 2005; Dilger et al., 2011).

\section{Discussion}

Our results demonstrate that $\mathrm{MD}$ promotes compensatory changes in the excitatory activity of dLGN cells. These alterations seem to be delayed compared with those in visual cortex (5-7 d vs 2 d) (Desai et al., 2002; Maffei et al., 2004), restricted to the monocular segment of dLGN (Coleman et al., 2009) that is innervated by the contralateral deprived eye, and involved the regulation of presynaptic glutamate release from corticothalamic terminals that synapse onto dLGN cells. These findings have the following implications. First, the visual thalamus and cortex share common forms of homeostatic plasticity. Second, the fact that the observed increase in mEPSC amplitude in the CDE region is coupled with a parallel increase in mEPSC frequency suggests that homeostatic plasticity involves a presynaptic mechanism. Finally, the increased probability of release at corticothalamic terminals strongly supports the hypothesis that the effects of MD in dLGN are driven by feedback excitation from the visual cortex.

The visual cortex has served as a model circuit system to study many forms of homeostatic plasticity, ranging from synaptic scaling to a modulation in excitatory and inhibitory circuitry (Turrigiano and Nelson, 2004; Turrigiano, 2008). Despite being mechanistically distinct, these alterations all serve the common goal of stabilizing neuronal activity in the face of degraded visual signaling. Thus, as a general rule, when synaptic activity is re- 
A
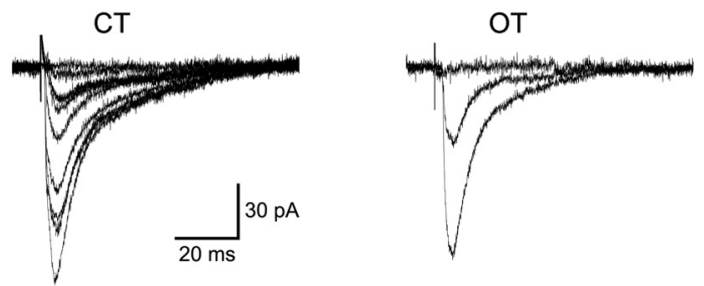

B
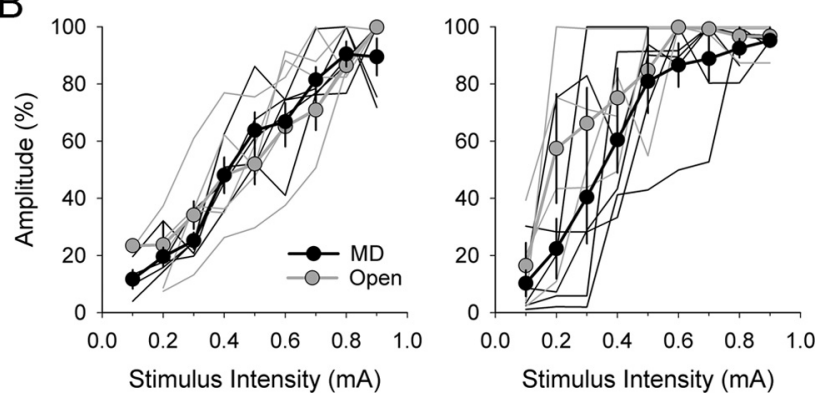

Figure 7. Input/output relations associated with $\mathrm{CT}$ and $0 \mathrm{~T}$ stimulation. $\boldsymbol{A}$, Examples of synaptic responses evoked by increasing levels of CT (left) and OT (right) stimulation for a relay neuron recorded in the deprived region of dLGN (Fig. 6). Responses are superimposed and each trace depicts the average of at least three responses for a given stimulus intensity recorded at a holding potential of $-70 \mathrm{mV}$. For CT stimulation, an incremental change of $0.1 \mathrm{~mA}$ (from 0.1 to $0.9 \mathrm{~mA}$ ) gave rise to graded changes in EPSC amplitude. By contrast, OT stimulation with the identical protocol yielded a step-like change in amplitude; one at 0.3-0.4 $\mathrm{mA}$ and another between 0.5 and $0.9 \mathrm{~mA}$. For clarity, only the responses at $0.4 \mathrm{~mA}$ and $0.7 \mathrm{~mA}$ are shown. $\boldsymbol{B}$, Plots of stimulus intensity by eEPSC amplitude for dLGN neurons recorded in deprived (black) and nondeprived (gray) eye regions for CT (left) and OT (right) stimulation. Thin lines represent the average responses (3-5 at a given intensity) for a single neuron and illustrate the relational change in amplitude as a function of stimulus intensity. Thick lines and symbols reflect the mean and \pm SE amplitude across different neurons. Note that for both deprived and open eye neurons, input/output curves for CT are graded and OT are step-like. CT MD, $n=5$ cells; CT open, $n=5$; OT MD, $n=6$; OT open, $n=4$.

duced below a basal level, compensatory changes are used to return the system to a functional range (Davis and Bezprozvanny, 2001; Burrone and Murthy, 2003, Turrigiano and Nelson, 2004). Our results in dLGN conform to this definition and demonstrate for the first time that homeostatic compensatory changes in excitatory activity can extend beyond the boundaries of neocortex to subcortical structures. Indeed, much like the reported increases in mEPSC amplitude that occur among deprived eye visual cortical neurons (Desai et al., 2002), relay cells in dLGN innervated by the deprived eye undergo a similar change. However, the mechanisms underlying these changes appear to be different. In the visual cortex, the increase in mEPSC amplitude is mediated by a postsynaptic accumulation of glutamate receptors, with no associated increase in presynaptic function (Desai et al., 2002; Wierenga et al., 2005; Gainey et al., 2009). In contrast, in dLGN, the increase in mEPSC amplitude after MD is accompanied by an increase in the mEPSC frequency, and, as our paired pulse experiments reveal, a higher probability of glutamate release. Currently, the signaling mechanisms that regulate the expression of presynaptic homeostatic plasticity remain a topic of intense investigation and it is unclear how presynaptic and postsynaptic forms interact (Turrigiano and Nelson, 2004; Turrigiano, 2008). Experiments performed on primary cultures of central neurons suggest both presynaptic and postsynaptic changes can be induced by experimentally reducing network activity and that the expression locus is related to maturational state of cultured synapses and/or the duration of imposed inactivity (Murthy et al., 2001; Wierenga et al., 2006).

Although our experiments were not designed to delineate the interplay between presynaptic and postsynaptic plasticity, they do implicate a presynaptic component that appears to arise from excitatory feedback connections that originate from layer VI of visual cortex. In fact, the homeostatic changes we observed in dLGN are in topographic register (Coleman et al., 2009) with those that have been reported in the visual cortex (Desai et al., 2002; Maffei et al., 2004, 2006; Maffei and Turrigiano, 2008). To be more specific, the CDE region of $\mathrm{dLGN}$ receives descending (deprived eye) input from the monocular region of visual cortex. Although it is known that large-scale changes in cortical excitability occur within the monocular region throughout layers II-IV after MD (Maffei et al., 2004, 2006; Maffei and Turrigiano, 2008), whether cells in layer VI, the source of corticofugal projections to the dLGN (Brumberg et al., 2003; Jacobs et al., 2007), undergo homeostatic compensation remains to be tested. Nonetheless, a complex array of reciprocal connections exists between layer VI and other cortical layers, which could support the modulation of excitatory and/or inhibitory activity (Burkhalter, 1989; Zarrinpar and Callaway, 2006; Thomson, 2010).

It is important to note that we cannot exclude the possibility that visual deprivation also affects activity at the retinogeniculate synapse since dark rearing alters the number of retinal inputs converging on a single dLGN cell (Hooks and Chen, 2006, 2008). Although our experiments do not provide a direct assessment of how retinogeniculate synapses respond to MD, our PPR results indicate that the probability of release at retinogeniculate terminals is not altered by MD. Perhaps, as others have shown in the visual cortex, different forms of deprivation lead to different outcomes (Fagiolini et al., 1994; Katz and Shatz, 1996; White et al., 2001; Hensch, 2005).

How can we account for the lack of homeostatic compensation in IDE region of dLGN? The simplest explanation is that this area receives descending input from the binocular region of visual cortex where the overall visual drive, although reduced, is in part compensated by the input from the open eye (Gordon and Stryker, 1996; Antonini et al., 1999; Frenkel and Bear, 2004). By contrast, the CDE region of dLGN, where homeostatic plasticity prevails, receives descending input exclusively from the monocular region of visual cortex. To date, cortical homeostatic plasticity induced by MD seems restricted to areas that receive input solely from the deprived eye, where the overall visual drive is maximally reduced (Desai et al., 2002; Maffei et al., 2004, 2006; Maffei and Turrigiano, 2008). Our results are in accord with these observations and further support the notion raised by others (Mrsic-Flogel et al., 2007) that the relative amount of visual drive received by the deprived eye is the critical element needed to trigger such plasticity.

In conclusion, our results suggest that in addition to a role in receptive field tuning and retinogeniculate signal modulation (Sherman, 2001; Briggs and Usrey, 2008), the corticothalamic pathway can convey compensatory changes in neuronal activity induced by altered visual experience. Such homeostatic feedback to thalamus may further influence how continued degraded retinal signals are processed and relayed to the visual cortex. Moreover, the fact that cortical and subcortical regions are constrained by similar homeostatic synaptic mechanisms may shed light on how the brain modulates and integrates activity to maintain neuronal network stability. 


\section{References}

Alexander GM, Fisher TL, Godwin DW (2006) Differential response dynamics of corticothalamic glutamatergic synapses in the lateral geniculate nucleus and thalamic reticular nucleus. Neuroscience 137:367-372.

Antonini A, Fagiolini M, Stryker MP (1999) Anatomical correlates of functional plasticity in mouse visual cortex. J Neurosci 19:4388-4406.

Bickford ME, Slusarczyk A, Dilger EK, Krahe TE, Kucuk C, Guido W (2010) Synaptic development of the mouse dorsal lateral geniculate nucleus. J Comp Neurol 518:622-635.

Briggs F, Usrey WM (2008) Emerging views of corticothalamic function. Curr Opin Neurobiol 18:403-407.

Brumberg JC, Hamzei-Sichani F, Yuste R (2003) Morphological and physiological characterization of layer VI corticofugal neurons of mouse primary visual cortex. J Neurophysiol 89:2854-2867.

Burkhalter A (1989) Intrinsic connections of rat primary visual cortex: laminar organization of axonal projections. J Comp Neurol 279:171-186.

Burrone J, Murthy VN (2003) Synaptic gain control and homeostasis. Curr Opin Neurobiol 13:560-567.

Chen C, Regehr WG (2000) Developmental remodeling of the retinogeniculate synapse. Neuron 28:955-966.

Chen C, Regehr WG (2003) Presynaptic modulation of the retinogeniculate synapse. J Neurosci 23:3130-3135.

Coleman JE, Law K, Bear MF (2009) Anatomical origins of ocular dominance in mouse primary visual cortex. Neuroscience 161:561-571.

Davis GW, Bezprozvanny I (2001) Maintaining the stability of neural function: A homeostatic hypothesis. Annu Rev Physiol 63:847-869.

Demas J, Eglen SJ, Wong RO (2003) Developmental loss of synchronous spontaneous activity in the mouse retina is independent of visual experience. J Neurosci 23:2851-2860.

Demas J, Sagdullaev BT, Green E, Jaubert-Miazza L, McCall MA, Gregg RG, Wong RO, Guido W (2006) Failure to maintain eye-specific segregation in nob, a mutant with abnormally patterned retinal activity. Neuron 50:247-259.

Desai NS, Cudmore RH, Nelson SB, Turrigiano GG (2002) Critical periods for experience-dependent synaptic scaling in visual cortex. Nat Neurosci 5:783-789.

Dilger EK, Shin HS, Guido W (2011) Requirements for synaptically evoked plateau potentials in relay cells of the dorsal lateral geniculate nucleus of the mouse. J Physiol 589:919-937.

Fagiolini M, Pizzorusso T, Berardi N, Domenici L, Maffei L (1994) Functional postnatal development of the rat primary visual cortex and the role of visual experience: dark rearing and monocular deprivation. Vision Res 34:709-720.

Frenkel MY, Bear MF (2004) How monocular deprivation shifts ocular dominance in visual cortex of young mice. Neuron 44:917-923.

Gainey MA, Hurvitz-Wolff JR, Lambo ME, Turrigiano GG (2009) Synaptic scaling requires the GluR2 subunit of the AMPA receptor. J Neurosci 29:6479-6489.

Golshani P, Warren RA, Jones EG (1998) Progression of change in NMDA, non-NMDA, and metabotropic glutamate receptor function at the developing corticothalamic synapse. J Neurophysiol 80:143-154.

Gordon JA, Stryker MP (1996) Experience-dependent plasticity of binocular responses in the primary visual cortex of the mouse. J Neurosci 16:3274-3286.

Govindaiah, Cox CL (2004) Synaptic activation of metabotropic glutamate receptors regulates dendritic outputs of thalamic interneurons. Neuron 41:611-623.

Granseth B, Lindström S (2003) Unitary EPSCs of cortiocgeniculate fibers in the rat dorsal lateral geniculate nucleus in vitro. J Neurophysiol 89:2952-2960.

Granseth B, Ahlstrand E, Lindström S (2002) Paired pulse facilitation of corticogeniculate EPSCs in the dorsal lateral geniculate nucleus of the rat investigated in vitro. J Physiol 544:477-486.

Hensch TK (2005) Critical period plasticity in local cortical circuits. Nat Rev Neurosci 6:877-888.

Hooks BM, Chen C (2006) Distinct roles for spontaneous and visual activity in remodeling of the retinogeniculate synapse. Neuron 52:281-291.

Hooks BM, Chen C (2008) Vision triggers an experience-dependent sensitive period at the retinogeniculate synapse. J Neurosci 28:4807-4817.
Hubel DH, Wiesel TN (1970) The period of susceptibility to the physiological effects of unilateral eye closure in kittens. J Physiol 206:419-436.

Huberman AD, Feller MB, Chapman B (2008) Mechanisms underlying development of visual maps and receptive fields. Annu Rev Neurosci 31:479-509.

Jacobs EC, Campagnoni C, Kampf K, Reyes SD, Kalra V, Handley V, Xie YY, Hong-Hu Y, Spreur V, Fisher RS, Campagnoni AT (2007) Visualization of corticofugal projections during early cortical development in a tauGFP-transgenic mouse. Eur J Neurosci 25:17-30.

Jaubert-Miazza L, Green E, Lo FS, Bui K, Mills J, Guido W (2005) Structural and functional composition of the developing retinogeniculate pathway in the mouse. Vis Neurosci 22:661-676.

Jones EG (2002) Thalamic circuitry and thalamocortical synchrony. Philos Trans R Soc Lond B Biol Sci 357:1659-1673.

Katz LC, Shatz CJ (1996) Synaptic activity and the construction of cortical circuits. Science 274:1133-1138.

Li J, Guido W, Bickford ME (2003) Two distinct types of corticothalamic EPSPs and their contribution to short-term plasticity. J Neurophysiol 90:3429-3440

Liu XB, Muñoz A, Jones EG (1998) Changes in subcellular localization of metabotropic glutamate receptor subtypes during postnatal development of mouse thalamus. J Comp Neurol 395:450-465.

Maffei A, Turrigiano GG (2008) Multiple modes of network homeostasis in visual cortical layer 2/3. J Neurosci 28:4377-4384.

Maffei A, Nelson SB, Turrigiano GG (2004) Selective reconfiguration of layer 4 visual cortical circuitry by visual deprivation. Nat Neurosci 7:1353-1359.

Maffei A, Nataraj K, Nelson SB, Turrigiano GG (2006) Potentiation of cortical inhibition by visual deprivation. Nature 443:81-84.

Mrsic-Flogel TD, Hofer SB, Ohki K, Reid RC, Bonhoeffer T, Hübener M (2007) Homeostatic regulation of eye-specific responses in visual cortex during ocular dominance plasticity. Neuron 54:961-972.

Murthy VN, Schikorski T, Stevens CF, Zhu Y (2001) Inactivity produces increases in neurotransmitter release and synapse size. Neuron 32:673-682.

Olson CR, Freeman RD (1980) Cumulative effect of brief daily periods of monocular vision on kitten striate cortex. Exp Brain Res 38:53-56.

Sherman SM (2001) Thalamic relay functions. Prog Brain Res 134:51-69.

Sherman SM, Guillery RW (2002) The role of the thalamus in the flow of information to the cortex. Philos Trans R Soc Lond B Biol Sci 357:1695-1708.

Smith GB, Heynen AJ, Bear MF (2009) Bidirectional synaptic mechanisms of ocular dominance plasticity in visual cortex. Philos Trans R Soc Lond B Biol Sci 364:357-367.

Smith SL, Trachtenberg JT (2007) Experience-dependent binocular competition in the visual cortex begins at eye opening. Nat Neurosci 10:370-375.

Thomson AM (2010) Neocortical layer 6, a review. Front Neuroanat 4:13.

Torborg CL, Feller MB (2005) Spontaneous patterned retinal activity and the refinement of retinal projections. Prog Neurobiol 76:213-235.

Turner JP, Salt TE (1998) Characterization of sensory and corticothalamic excitatory inputs to rat thalamocortical neurones in vitro. J Physiol 510:829-843.

Turrigiano GG (2008) The self-tuning neuron: synaptic scaling of excitatory synapses. Cell 135:422-435.

Turrigiano GG, Nelson SB (2004) Homeostatic plasticity in the developing nervous system. Nat Rev Neurosci 5:97-107.

Turrigiano GG, Leslie KR, Desai NS, Rutherford LC, Nelson SB (1998) Activity-dependent scaling of quantal amplitude in neocortical neurons. Nature 391:892-896.

White LE, Coppola DM, Fitzpatrick D (2001) The contribution of sensory experience to the maturation of orientation selectivity in ferret visual cortex. Nature 411:1049-1052.

Wierenga CJ, Ibata K, Turrigiano GG (2005) Postsynaptic expression of homeostatic plasticity at neocortical synapses. J Neurosci 25:2895-2905.

Wierenga CJ, Walsh MF, Turrigiano GG (2006) Temporal regulation of the expression locus of homeostatic plasticity. J Neurophysiol 96:2127-2133.

Williams SR, Mitchell SJ (2008) Direct measurement of somatic voltage clamp errors in central neurons. Nat Neurosci 11:790-798.

Zarrinpar A, Callaway EM (2006) Local connections to specific types of layer 6 neurons in the rat visual cortex. J Neurophysiol 95:1751-1761. 